\title{
Risk factors for situs defects and congenital heart disease in primary ciliary dyskinesia
}

Sunayna Best, ${ }^{1,2}$ Amelia Shoemark, ${ }^{2,3}$ Bruna Rubbo, ${ }^{4,5,6}$ Mitali P Patel, ${ }^{1}$ Mahmoud R Fassad, ${ }^{1,7}$ Mellisa Dixon, ${ }^{2}$ Andrew V Rogers, ${ }^{2,8}$ Robert A Hirst, ${ }^{9}$ Andrew Rutman, ${ }^{9}$ Sarah Ollosson, ${ }^{2}$ Claire L Jackson, 4,5,6 Patricia Goggin, 4,5,6 Simon Thomas, ${ }^{10,11}$ Reuben Pengelly, ${ }^{10}$ Thomas Cullup, $^{12}$ Eleni Pissaridou, ${ }^{13}$ Jane Hayward, ${ }^{1,12}$ Alexandros Onoufriadis, ${ }^{14}$ Christopher O'Callaghan, ${ }^{9,15}$ Michael R Loebinger, ${ }_{1}^{8}$ Robert Wilson, ${ }^{8}$ Eddie MK Chung, ${ }^{13}$ Priti Kenia, $^{16}$ Victoria L Doughty, ${ }^{17}$ Julene S Carvalho, ${ }^{17,18,19}$ Jane S Lucas, ${ }^{4,5,6}$ Hannah M Mitchison, ${ }^{1}$ Claire $\mathrm{Hogg}^{2}$

- Additional material is published online only. To view please visit the journal online (http://dx.doi.org/10.1136/ thoraxjnl-2018-212104).

For numbered affiliations see end of article.

Correspondence to Dr Hannah M Mitchison, Department of Genetics and Genomic Medicine, UCL Great Ormond Street Institute of Child Health, London WC1N 1EH, UK; h.mitchison@ucl.ac.uk

HMM and $\mathrm{CH}$ are shared senior authorship.

Received 23 May 2018 Revised 4 July 2018 Accepted 23 July 2018 Published Online First 30 August 2018

Check for updates

(C) Author(s) (or their employer(s)) 2019. No commercial re-use. See rights and permissions. Published by BMJ.

To cite: Best $S$, Shoemark $A$, Rubbo $\mathrm{B}$, et al. Thorax

2019:74:203-205

\section{ABSTRACT}

Primary ciliary dyskinesia (PCD) is associated with abnormal organ positioning (situs) and congenital heart disease (CHD). This study investigated genotypephenotype associations in PCD to facilitate risk predictions for cardiac and laterality defects. This retrospective cohort study of 389 UK patients with PCD found $51 \%$ had abnormal situs and $25 \%$ had CHD and/ or laterality defects other than situs inversus totalis. Patients with biallelic mutations in a subset of nine PCD genes had normal situs. Patients with consanguineous parents had higher odds of situs abnormalities than patients with non-consanguineous parents. Patients with abnormal situs had higher odds of CHD and/or laterality defects.

\section{INTRODUCTION}

Primary ciliary dyskinesia (PCD) arises from the dysfunction of motile cilia and has an estimated prevalence of one in 10000 births. Abnormal cilia structure or function leads to organ laterality defects in approximately half of the patients with PCD. ${ }^{12}$ This arises from impaired function of motile cilia in the embryonic left-right organiser (node), ${ }^{3}$ causing random assignment of thoracoabdominal orientation. Two past studies investigated rates of laterality defects and CHD in PCD, with combined results showing $3.5 \%-6 \%$ of patients with PCD had a cardiovascular malformation. $^{4-6}$

To date, over 35 identified PCD genes are reported to account for about $70 \%$ of screened, well-diagnosed cases. ${ }^{7}$ Some PCD gene mutations are never associated with situs abnormalities, connected to a lack of functional requirement for their encoded proteins in the embryonic node. ${ }^{78}$

It is well established that cilia motility plays a major role in laterality determination, ${ }^{3}$ but much remains unknown about the clinical and genetic risk factors for situs defects and CHD pathogenesis in motile ciliopathy disorders.

\section{METHODS}

This is a retrospective cohort study of 389 patients seen in specialist UK clinics with a diagnosis of PCD according to European Respiratory Society (ERS) guidelines. ${ }^{9}$ Full details are described in the online Supplementary methods.

Situs was classified as: (1) situs solitus (SS), defined as normal organ arrangement, (2) situs inversus totalis (SIT), defined as mirror image arrangement of all organs or (3) situs ambiguus (SA), defined as any abnormal arrangement that was not SS or SIT. A two-stage system was used for organ defect classification (online Supplementary table S1). Statistical analysis focused on associations between clinical and genetic factors and two main outcomes: situs abnormality and CHD and/or structural laterality defects. Analysis was performed using Fisher's exact test and univariate and multivariable logistic regression modelling.

Genes were assigned to two groups (A and B) according to whether they have previously been associated to situs abnormalities in the literature (online Supplementary table S2): Group A genes associated with situs abnormalities and Group $B$ genes not previously associated with situs abnormalities.

\section{RESULTS}

The clinical data and genetic test results available for analysis in the 389 confirmed patients with PCD in the study are shown in the online Supplementary figure S1, along with the details of CHD and laterality defects identified (online Supplementary table S3) and full results of statistical regression modelling (online Supplementary table S4).

\section{Situs abnormalities}

$49.2 \%$ of patients had SS, $41.9 \%$ had SIT and $8.9 \%$ had SA. The distribution of normal and abnormal situs arrangements was assessed for each of 27 PCD genes found to be mutated in the 199 patients for whom both situs was determined and genetics solved. Notably, for 18 genes, patients with biallelic mutations had normal or abnormal situs, while patients with biallelic mutations in the other nine genes all had normal situs (figure 1). This difference in frequency of situs abnormality between patients with mutations in Group B versus Group A genes (0/38 vs 98/161, respectively) highlights a significant association between situs abnormality 


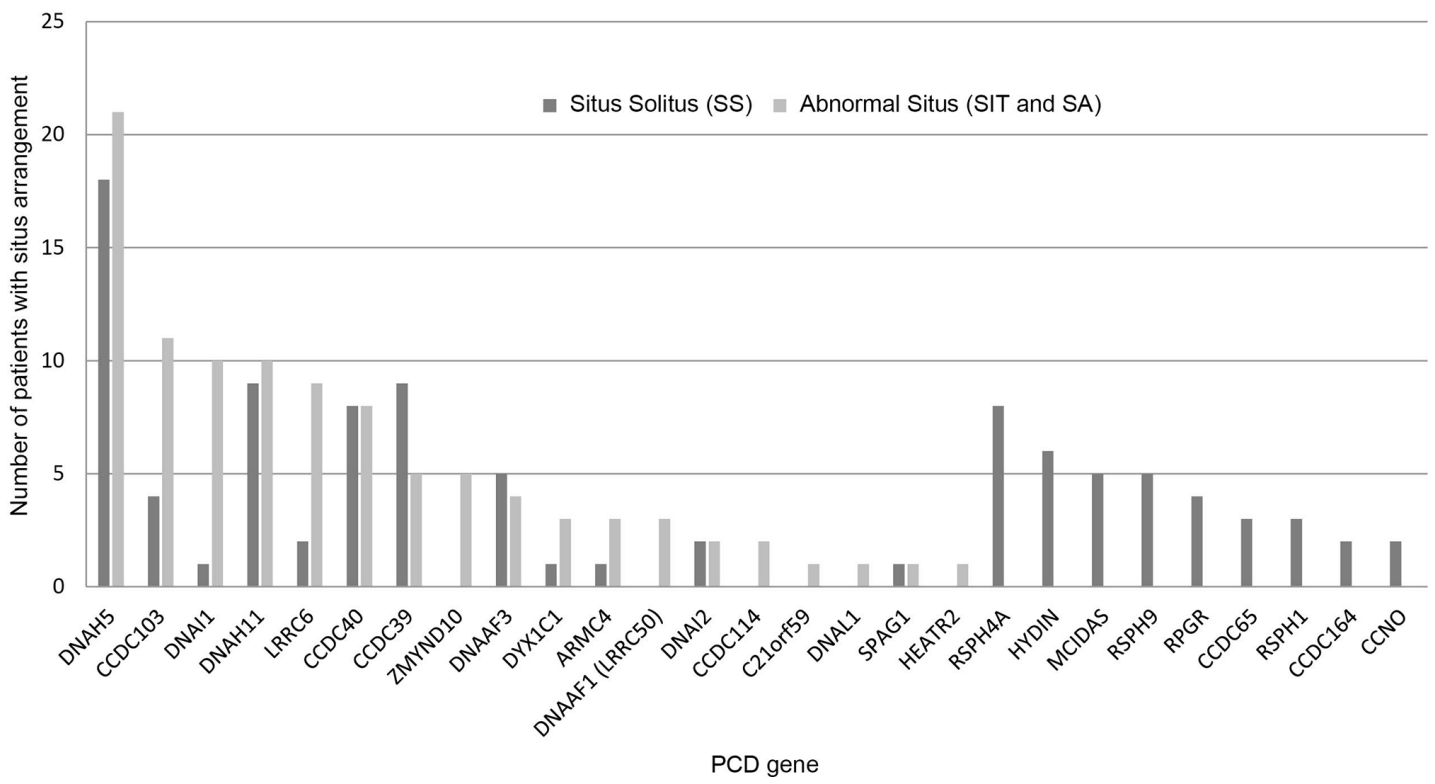

Figure 1 Situs distribution observed for each primary ciliary dyskinesia (PCD) gene identified to have pathogenic mutations, among the genetically solved cohort. This shows the number of patients with normal situs (situs solitus (SS)) and abnormal situs (situs inversus totalis (SIT) and situs ambiguus (SA)) for each known PCD gene ( $n=27)$ among the 199 patients identified to have biallelic mutations in whom situs was known. No abnormal situs is detected in patients with mutations in nine genes, all from group B: CCDC164 (DRC1), CCDC65 (DRC2), CCNO, HYDIN, MCIDAS, RPGR, RSPH1, RSPH4A and RSPH9.

in our cohort and the literature evidence for situs abnormality $(\mathrm{p}<0.001$, Fisher's exact test) (online Supplementary table S4, outcome 1).

Parental consanguinity, ethnicity and functional gene effect were evaluated as potential risk factors for situs abnormality. Only parental consanguinity was found to be significantly associated with situs abnormality (online Supplementary table S4, outcome 1). Univariate modelling suggests there is a $77.2 \%$ increase in the odds of situs abnormality for patients with consanguineous parents compared with those with non-consanguineous parents (OR $1.77, \mathrm{p}=0.02,95 \%$ CI 1.09 to 2.88 ).

\section{CHDs and structural laterality defects}

$25.2 \%$ of patients had CHD and/or laterality defects other than SIT. The prevalence of CHD and/or laterality defects according to situs group is shown in figure 2 .

In a risk factor model, only situs abnormality was found to be significantly associated with the presence of CHD and/or laterality defects other than SIT (online Supplementary table S4, outcome 2). The univariate model suggests that there is an $98 \%$ increase in the odds of having CHD and/or laterality defects other than SIT for patients with abnormal situs, compared with the group of patients with normal situs (OR 7.98, p<0.001, 95\% CI 3.57 to 17.83).

\section{DISCUSSION}

This is the first study investigating situs and laterality defects in patients with PCD from the UK. Compared with previously published studies, ${ }^{56}$ there is a similar situs distribution, but we identify at least three times higher prevalence of CHD in this PCD population (17.1\% of cases). The observed prevalence of laterality defects other than SIT (14.1\%) was also high.

The identified prevalence of CHD and laterality defects

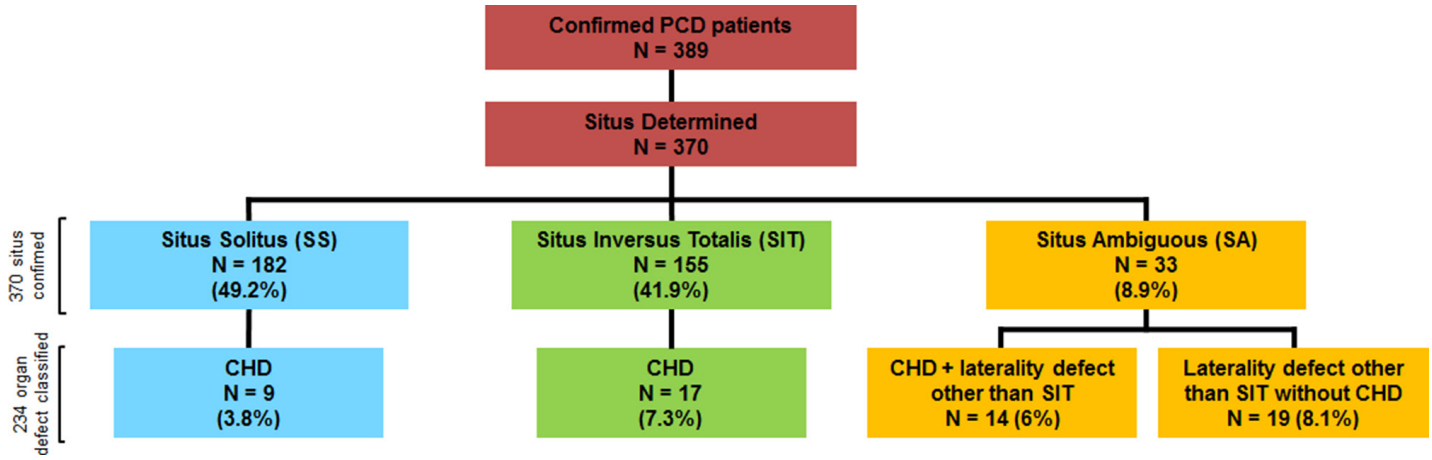

Figure 2 Distribution of situs arrangements among the patients with primary ciliary dyskinesia (PCD) and a breakdown of congenital heart disease (CHD) and other laterality defects in each situs group. The number of patients in each category is given. The percentage of patients in each situs group (situs solitus (SS), situs inversus totalis (SIT), situs ambiguus (SA)) was calculated from the total number of patients in whom situs was determined $(n=370)$. The percentage of patients with each category of CHD and/or laterality defect other than SIT was calculated from the total number of patients who fulfilled criteria for organ defect classification $(n=234)$. 
must be interpreted carefully given the difference in classification systems used in the previous studies. We chose to classify according to severity, deciding this was most important for patient care. International consensus on nomenclature and classification for situs and laterality defects would improve comparison between research studies. For completeness, we did also classify our cohort using the same modified Botto et al system ${ }^{10}$ as used by previous studies ${ }^{4-6}$ (online Supplementary table S3).

The higher observed prevalence of laterality and cardiac defects among our patients compared to levels reported previously could be due to a difference in populations. We have an ethnically diverse cohort, with a high proportion with consanguineous parents, who may have more severe disease phenotypes.

A limitation to this study was variation in the availability of detailed imaging data among patients. We acknowledge that a selection bias is possible for patients with detailed imaging towards those more likely to have $\mathrm{CHD} /$ other laterality defects based on their history or clinical examination.

Given the higher than anticipated prevalence of cardiac and laterality defects identified in this study, we recommend that all patients diagnosed with PCD have a cardiac echocardiogram and abdominal ultrasound. These are simple, harmless and inexpensive tests. Many of the structural laterality defects are clinically actionable, and so are important to detect.

Our study affirms the importance of genetic predisposition to laterality defects in PCD, since a subset of PCD genes were clearly not associated with situs problems.

In summary, this study illustrates that improved knowledge about genotype-phenotype correlations in PCD may facilitate risk predictions for $\mathrm{CHD}$ and laterality defects as well as other clinical consequences, allowing for early detection and treatment.

\section{Author affiliations}

${ }^{1}$ Genetics and Genomic Medicine, University College London (UCL) Great Ormond Street Institute of Child Health, London, UK

${ }^{2}$ PCD Diagnostic Team, Department of Paediatric Respiratory Medicine, Royal Brompton and Harefield NHS Trust, London, UK

${ }^{3}$ School of Medicine, University of Dundee, Dundee, UK

${ }^{4}$ Primary Ciliary Dyskinesia Centre, University Hospital Southampton NHS Foundation Trust, Southampton, UK

${ }^{5}$ Clinical and Experimental Sciences Academic Unit, Faculty of Medicine, University of Southampton, Southampton, UK

${ }^{6}$ NIHR Southampton Biomedical Research Centre, University of Southampton and University Hospital Southampton NHS Foundation Trust, Southampton, UK ${ }^{7}$ Human Genetics Department, Alexandria University, Alexandria, Egypt

${ }^{8}$ Host Defence Unit, Royal Brompton and Harefield NHS Trust, London, UK

${ }^{9}$ Department of Infection, Immunity and Inflammation, Centre for PCD Diagnosis and Research, RKCSB, University of Leicester, Leicester, UK

${ }^{10}$ Human Genetics and Genomic Medicine, Faculty of Medicine, University of

Southampton, Southampton, UK

${ }^{11}$ Wessex Regional Genetics Laboratory, Salisbury NHS Foundation Trust, Salisbury

District Hospital, Salisbury, UK

${ }^{12}$ North East Thames Regional Genetics Service, Great Ormond Street Hospital for

Children, London, UK

${ }^{13}$ Population, Policy and Practice Programme, University College London (UCL) Great

Ormond Street Institute of Child Health, London, UK

${ }^{14}$ Division of Genetics and Molecular Medicine, Department of Medical and

Molecular Genetics, King's College London School of Medicine, Guy's Hospital, London, UK

${ }^{15}$ Department of Respiratory, Critical Care and Anaesthesia, University College London (UCL) Great Ormond Street Institute of Child Health, London, UK

${ }^{16}$ Department of Respiratory Paediatrics, Birmingham Children's Hospital NHS

Foundation Trust, Birmingham, UK

${ }^{17}$ Brompton Centre for Fetal Cardiology, Royal Brompton and Harefield NHS Trust, London, UK

${ }^{18}$ Fetal Medicine Unit, St George's University Hospitals NHS Foundation Trust,
London, UK

${ }^{19}$ Molecular and Clinical Sciences Research Institute, St George's, University of London, London, UK

Acknowledgements We are very grateful to the families with PCD who participated in this study and to the UK PCD Family Support Group for their support. We acknowledge the PCD diagnostic and clinical teams for their care of the patients and their contribution towards the phenotyping, particularly Dr Woolf Walker, Dr Siobhan Carr and Professor Andrew Bush. We would like to thank Dr Edite Goncalves and Dr Christos Kokkinakis for partial data collection on cardiac status at the Royal Brompton Hospital. We are grateful to Hywel J. Williams, Juliet Scully, Miriam Schmidts, the UCL Centre for Translational Omics (GOSgene), Lucy Jenkins and Christopher Boustred for experimental support and data analysis.

Contributors SB compiled, managed and analysed the clinical and genetic data. $S B, A S$ and $B R$ searched clinical records and compiled the clinical data. SB, MPP, MRF, $S T, R P, T C, J H$ and $A O$ performed genetic analyses. AS, MD, AVR, RAH, AR, SO, CLJ and PG performed clinical cilia functional testing and imaging studies. EP advised on and performed statistical analysis. CO'C, MRL, RW, EC, PK, JSC, JSL and CH contributed to clinical analysis and data management. VLD and JSC contributed to cardiac data management and interpretation. SB, AS, JSC, HMM and CH wrote the manuscript. HMM, JSC and CH designed the project and are responsible for the overall content. All authors reviewed the data, revised the manuscript for logical content and approved the final version.

Funding This research is supported by the BEAT-PCD: Better Evidence to Advance Therapeutic Options for PCD network (COST Action 1407). Work at the Royal Brompton Hospital was partially supported by the European Society of Cardiology. SB was supported by an Academic Clinical Fellowship funded by the National Institute of Health Research (NIHR) and Imperial College London Biomedical Research Centre (BRC). Work in Southampton is supported by NIHR Respiratory BRC and NIHR Wellcome Trust Clinical Research Facility. Work by AS was independent research funded by a postdoctoral research fellowship from the NIHR and Health Education England. EP, CO'C and HMM are supported by the NIHR Biomedical Research Centre at Great Ormond Street Hospital for Children NHS Foundation Trust and University College London. HMM acknowledges grants from Action Medical Research (GN2101), Newlife Foundation (10-11/15) and the Great Ormond Street Hospital Children's Charity.

Disclaimer The views expressed in the submitted article are the authors' own and not an official position of the institution or funder.

Competing interests None declared.

Patient consent Not required.

Ethics approval London Bloomsbury Research Ethics Committee.

Provenance and peer review Not commissioned; externally peer reviewed.

Data sharing statement No additional data.

\section{REFERENCES}

1 Lucas JS, Burgess A, Mitchison HM, et al. Diagnosis and management of primary ciliary dyskinesia. Arch Dis Child 2014;99:850-6.

2 Leigh MW, Pittman JE, Carson JL, et al. Clinical and genetic aspects of primary ciliary dyskinesia/Kartagener syndrome. Genet Med 2009;11:473-87.

3 Pennekamp P, Menchen T, Dworniczak B, et al. Situs inversus and ciliary abnormalities: 20 years later. what is the connection? Cilia 201541.

4 Harrison MJ, Shapiro AJ, Kennedy MP. Congenital heart disease and primary ciliary dyskinesia. Paediatr Respir Rev 2016;18:25-32.

5 Shapiro AJ, Davis SD, Ferkol T, et al. Laterality defects other than situs inversus totalis in primary ciliary dyskinesia: insights into situs ambiguus and heterotaxy. Chest 2014;146:1176-86.

6 Kennedy MP, Omran H, Leigh MW, et al. Congenital heart disease and other heterotaxic defects in a large cohort of patients with primary ciliary dyskinesia. Circulation 2007;115:2814-21.

7 Mitchison HM, Valente EM. Motile and non-motile cilia in human pathology: from function to phenotypes. J Pathol 2017;241:294-309.

8 Knowles MR, Daniels LA, Davis SD, et al. Primary ciliary dyskinesia. Recent advances in diagnostics, genetics, and characterization of clinical disease. Am J Respir Crit Care Med 2013;188:913-22.

9 Lucas JS, Barbato A, Collins SA, et al. European Respiratory Society guidelines for the diagnosis of primary ciliary dyskinesia. Eur Respir J 2017;49.

10 Botto LD, Lin AE, Riehle-Colarusso T, et al. Seeking causes: classifying and evaluating congenital heart defects in etiologic studies. Birth Defects Res A Clin Mol Teratol 2007:79:714-27. 